\title{
Survival Outcomes and Prognostic Factors of Borderline Ovarian Tumors
}

\section{Supreechaya Phansenee, Charuwan Tantipalakorn, Tanarat Muangmool, Kittipat Charoenkwan, Prapaporn Suprasert, Jatupol Srisomboon}

Department of Obstetrics and Gynecology, Faculty of Medicine, Chiang Mai University, Thailand.

\begin{abstract}
Background and Objective: Borderline ovarian tumor (BOT) is a distinct but heterogeneous group of tumors defined by histopathology as atypical epithelial proliferation without stromal invasion. Women with BOT are usually younger than those with invasive carcinoma. This study aimed to evaluate the survival outcomes and prognostic factors of patients with BOT in a single institute in the northern region of Thailand. Methods: The medical records of patients with BOT who were treated at Chiang Mai University Hospital between January 1, 2008 and December 31, 2019 were reviewed. The survival outcomes were analyzed by the Kaplan-Meier method and prognostic factors were analyzed by univariate and multivariate approaches. Results: 168 patients with BOT were enrolled in the study. The median age was 48.8 years. At the median follow-up time of 25.4 months, 9 patients $(5.3 \%)$ developed recurrence and $2(1.1 \%)$ experienced progression to invasive carcinoma. The 5 -year progression-free survival (PFS) and overall survival were $93.2 \%$ and $97.4 \%$, respectively. By univariate analysis, advanced stage $(\mathrm{p}=0.02)$, tumor size smaller than $10 \mathrm{~cm}(\mathrm{p}=0.03)$, conservative surgery $(\mathrm{p}=0.03)$, and bilateral tumors $(\mathrm{p}=0.07)$ were significantly associated with worse PFS. Hysterectomy was a protective factor for recurrence with the hazard ratio of $0.09(95 \% \mathrm{CI}, 0.01-0.77 ; \mathrm{p}=0.03)$. Cell types, pelvic lymphadenectomy, micro-invasion, and non-invasive peritoneal implant did not significantly affect PFS. By multivariate analysis, early stage $(\mathrm{p}=0.01)$, tumor size larger than $10 \mathrm{~cm}(\mathrm{p}=0.04)$, and hysterectomy $(\mathrm{p}=0.03)$ were significantly associated with better survival. Conclusion: Patients with BOT had excellent survival outcomes. Tumor size of larger than $10 \mathrm{~cm}$, early-stage disease, and hysterectomy were significant prognostic factors for better survival outcomes. Conservative surgery should be offered to patients who desire to preserve future fertility and long-term follow-up is needed to assure recurrence - free.
\end{abstract}

Keywords: Ovarian tumor- borderline- survival- prognostic factor

\section{Introduction}

Borderline ovarian tumor (BOT) or atypical proliferative tumor is once known as a tumor of low malignant potential (LMP). The diagnosis of BOT is based on pathological findings of atypical proliferation as seen in epithelial ovarian cancer, without stromal invasion. Occasionally, a non-invasive peritoneal implant could be found in patients with BOT. According to WHO classification in2014, a micro-invasion has been reported, in which the stromal invasion is observed less than $5 \mathrm{~mm}$ in the greatest linear dimension [1].
BOT is the tumor of a young age group and one-third of them are under 40 years of age $[2,3]$. Most patients with BOT present at an early stage, and the prognosis is generally favorable. The pathology of BOT is classified into 6 subtypes in which the most prevalent type is serous followed by mucinous, whereas endometrioid, clear cell, Brenner, and mixed sero-mucinous types are rarely found $[1,4]$.

Treatment for BOT depends on fertility desire. For patients who no longer need to preserve future fertility, complete surgical resection is usually performed as the

\section{Corresponding Author:}

Dr. Jatupol Srisomboon

Department of Obstetrics and Gynecology, Faculty of Medicine, Chiang Mai University, Thailand.

Email: jatupol1957@hotmail.com 
same standard treatment for epithelial ovarian cancer. A certain study noted that in BOT, pelvic lymph node dissection is not necessary to perform as there is no benefit in improving the survival rate [5]. For patients who need to preserve future childbearing, fertility-sparing surgery should be offered by performing unilateral salpingo-oophorectomy ( $\mathrm{SO}$ ), sparing uterus, and contralateral normal ovary. Ovarian cystectomy could be performed in the setting of patients who have undergone contralateral SO or tumors involve bilateral ovaries without minimizing pregnancy rate [6]. However, the recurrence rate in patients undergoing ovarian cystectomy was significantly higher than those undergoing SO [7]. Adjuvant chemotherapy remains a controversial issue for BOT [4]. One study reported that chemotherapy was associated with worse survival outcomes [8].

In general, the recurrence rate of BOT patients is quite low with adequate follow up protocol. However, several factors have been identified as an increased risk of recurrence, i.e., conservative surgery, suboptimal surgery, non-invasive peritoneal implant, micro-invasion, and micropapillary architecture $[9,10]$.

This study was conducted to evaluate the survival outcomes and to identify the prognostic factors of patients with BOT undergoing surgical treatment at Chiang Mai University Hospital in the northern region of Thailand.

\section{Materials and Methods}

After approval of the Research Ethics Committee of Chiang Mai University Hospital (CMUH), the data were collected by retrospective review from January 1, 2008, to December 31, 2019 at CMUH, Chiang Mai, Thailand. The study population was women with BOT diagnosed from pathological examination and received treatment at the CMUH. The pathology slides were reviewed by gynecologic pathologists if the patients were referred from other hospitals.

Patients with malignant ovarian tumors, other concurrent malignancy, and incomplete medical records were excluded. The medical records including demographic data, preoperative tumor markers (CA125, CA19-9, and CEA), pathological characteristics (cell subtypes, non-invasive implants, micro-invasion) were retrieved from the electronic database of gynecologic oncology units.

The surgical procedure varied depending on the surgeon's discretion, the frozen section results, and intraoperative findings. In cases with fertility desire, conservative surgery was carried out by performing unilateral SO, preserving contralateral normal ovary without hysterectomy. Hysterectomy with bilateral SO was defined as definitive surgery, while omentectomy, lymphadenectomy, and peritoneal biopsy were defined as surgical staging procedures.

All patients were followed every 3 months during the first year of surgery, every 4 months in year 2 , every 6 months in year 3-5, and then yearly after year 5. Surveillance included clinical examination, pelvic examination, and tumor markers. Pelvic ultrasound was performed instead in those who were not able to undergo pelvic examination. CT scans or MRIs were used in patients with suspected recurrent diseases. Progression-free survival was the duration since the surgery to the presence of recurrent diseases and overall survival was the duration since the surgery until death.

Statistical analyses were performed using STATA version 15 (College Station, TX: StataCorp LLC.). Descriptive statistics were reported by median (interquartile range) for the continuous variables and frequency (percentage) for categorical variables. Univariate and multivariate analyses were used to identify prognostic factors in the progression-free survival of BOT. The factors with a $p$-value of $\leq 0.10$ in the univariate analysis were further analyzed in multivariate logistic regression models. The survival function was estimated by the Kaplan-Meier method and distribution for each group was compared by the log-rank test. A p-value of $\leq 0.05$ was considered statistically significant.

\section{Results}

During the study period, 197 patients with BOT were identified in the database. Eighteen patients were excluded after pathological review, i.e., invasive epithelial ovarian cancer (7), benign mucinous cystadenoma (1), concurrent cancer (8), mucinous BOT from gastro-intestinal malignancy metastases (2). Six patients had incomplete medical records and 5 were lost to follow-up after the operation. Therefore, 168 patients were eligible for analysis. The clinical characteristics are shown in Table 1. Among 168 patients, 91 (54\%) patients were younger than 50 years old. Elevated serum CA125 (>35 U/mL) was noted in $90(62 \%)$ patients. High serum CA19-9 $(>39 \mathrm{U} / \mathrm{mL})$ and serum CEA $(>5.2 \mathrm{ng} / \mathrm{ml})$ were found in $52(38 \%)$ and $30(22 \%)$ patients, respectively. Definitive surgery was performed in 123 patients $(73 \%)$, while conservative surgery was carried out in 45 (27\%). Pelvic and para-aortic lymphadenectomy was performed in 63 (38\%) and 24 (14\%) patients, respectively. Adjuvant chemotherapy with paclitaxel with carboplatin was administered in 15 patients $(9 \%)$.

Tumor characteristics are shown in Table 2. The most common cell type of BOT was mucinous $(70 \%)$ followed by serous $(24 \%)$ and endometrioid (5\%). One hundred and fifty-six $(93 \%)$ patients had stage I BOT while the remaining 12 (7\%) had stage II and III diseases. Eighty-two percent of the patients had tumor size larger than $10 \mathrm{~cm}$. The average tumor size was $17.3 \mathrm{~cm}$. Two of 63 patients (3\%) had pelvic lymph node metastasis where none of the 24 patients had para-aortic lymph node metastasis. From the histological review, 31 patients had microinvasive peritoneal implants and 16 had non-invasive peritoneal implants.

Oncologic outcomes are shown in Table 3. During the median follow-up time of 25.4 months, only 9 patients $(5.5 \%)$ developed disease recurrence with an estimated 5-year progression-free survival (PFS) of 93.2\%. Among these recurrences, 6 and 3 patients had prior stage I and stage II -III, respectively. Of the 9 patients with 
Table 1. Clinical Characteristics of 168 Patients with Borderline Ovarian Tumor

\begin{tabular}{lc}
\hline Characteristics & $\begin{array}{c}\text { Number (\%) or Median } \\
\text { (Range)* }\end{array}$ \\
\hline Age (Years) & $48.4(10.5-79.1)^{*}$ \\
$<50$ & $91(54)$ \\
$\geq 50$ & $77(46)$ \\
CA $125(\mathrm{U} / \mathrm{mL})(\mathrm{n}=143)$ & $52(26.3-143.3)$ \\
$<35$ & $55(38)$ \\
$\geq 35$ & $90(62)$ \\
CA $19-9(\mathrm{U} / \mathrm{mL})(\mathrm{n}=137)$ & $22.6(6.6-119.1)$ \\
$<39$ & $86(62)$ \\
$\geq 39$ & $52(38)$ \\
CEA (ng/ml) (n=136) & $2.0(1.1-4.6)$ \\
$<5.2$ & $107(78)$ \\
$\geq 5.2$ & $30(22)$ \\
Primary treatment & \\
Definitive surgery & $123(73)$ \\
Conservative surgery & $45(27)$ \\
Pelvic lymphadenectomy & $105(62)$ \\
No & $63(38)$ \\
Yes & \\
Para-aortic lymphadenectomy & \\
No & \\
Yes & \\
Adjuvant chemotherapy & \\
No & \\
Yes & \\
\hline
\end{tabular}

*Descriptive statistics were reported by median (interquartile range) for the continuous variables and frequency (percentage) for categorical variables; * Age was described by median (minimum-maximum)

recurrences, 4 were serous and mucinous types each, the other 1 was endometrioid type. 8 patients had single site recurrence and 1 had recurrence at multiple sites. Among 9 patients with recurrences, 6 were treated by conservative surgical excision, while the remaining 3 underwent definitive surgery. Bilateral BOT was found in 3 patients and only 1 underwent lymphadenectomy. Two patients developed invasive tumor recurrence. One with pelvic lymph node recurrence of endometrioid adenocarcinoma was treated with surgical excision. The remaining 1 with supraclavicular lymph node recurrence of serous adenocarcinoma was treated with chemotherapy.

Five-year overall survival of the 168 BOT patients was $97.4 \%$ with 7 deaths. One patient with invasive endometrioid adenocarcinoma recurrence had tumor invasion at rectum and ureters causing severe hemorrhage and renal insufficiency. Five patients died from other underlying diseases and the remaining 1 died from cholangiocarcinoma occurring 6 years after the detection of BOT.

Prognostic factors were analyzed using the univariate and multivariate analyses as shown in Table 4. In univariate analysis, PFS was significantly worse in patients with advanced stage $(P=0.02)$, tumor size $\leq 10 \mathrm{~cm}$ $(p=0.03)$, conservative surgery $(p=0.03)$, and bilateral tumors $(\mathrm{p}=0.07)$. Interestingly, hysterectomy was a protective factor with a hazard ratio (HR) of 0.09 (95\% CI $0.01-0.77, p=0.03)$. Cell types, pelvic lymphadenectomy, micro-invasion, and non-invasive peritoneal implants were not significantly associated with survival outcomes. In multivariate analysis, early stage, tumor size $>10 \mathrm{~cm}$, and hysterectomy were significantly associated with better survival.

\section{Discussion}

This study showed that patients with BOT had an excellent prognosis. More than $90 \%$ of the patients were detected in the early stage similar to the previous report [11]. The most common cell subtype was mucinous accounting for $70 \%$ in our study resembling many studies in East Asia [12-14]. In contrast, serous BOT was more commonly found in North America, Europe, and Middle East. The precise cause of the differences in the histologic distribution in each region remains elusive [15].

Table 2. Tumor Characteristics of 168 Patients with Borderline Ovarian Tumor

\begin{tabular}{|c|c|}
\hline Variables & Number of patients $(\%)$ \\
\hline \multicolumn{2}{|l|}{ Cell type } \\
\hline Endometrioid & $8(5)$ \\
\hline Mucinous & $118(70)$ \\
\hline Serous & $40(24)$ \\
\hline Mixed & $2(1)$ \\
\hline \multicolumn{2}{|l|}{ Stage } \\
\hline I & $156(93)$ \\
\hline II-III & $12(7)$ \\
\hline \multicolumn{2}{|c|}{ Tumor size $(\mathrm{cm})(\mathrm{n}=166)$} \\
\hline$\leq 10$ & $30(18)$ \\
\hline$>10$ & $136(82)$ \\
\hline \multicolumn{2}{|c|}{ Pelvic lymph node involvement $(\mathrm{n}=63)$} \\
\hline No & $61(97)$ \\
\hline Yes & $2(3)$ \\
\hline \multicolumn{2}{|c|}{ Para-aortic lymph node involvement $(\mathrm{n}=24)$} \\
\hline No & $24(100)$ \\
\hline Yes & $0(0)$ \\
\hline \multicolumn{2}{|l|}{ Micro-invasion } \\
\hline No & $137(82)$ \\
\hline Yes & $31(19)$ \\
\hline \multicolumn{2}{|c|}{ Non-invasive implant } \\
\hline No & $152(91)$ \\
\hline Yes & $16(10)$ \\
\hline \multicolumn{2}{|l|}{ Laterality } \\
\hline Unilateral & $151(90)$ \\
\hline Bilateral & $17(10)$ \\
\hline
\end{tabular}

Descriptive statistics were reported by frequency (percentage) for categorical variables. 
Table 3. Oncological Outcomes of Borderline Ovarian Tumor Patients ( $\left.\mathrm{N}=161^{*}\right)$

\begin{tabular}{lc}
\hline Characteristics & Number of patients (\%) or Median (Range)* \\
\hline Follow-up time (months) & $25.4(9.9-56.3)$ \\
Alive & $156(96)$ \\
Recurrence & $9(5.5)$ \\
$\quad$ Recurrence with borderline tumor & $7(4.3)$ \\
$\quad$ Recurrence with invasive carcinoma & $2(1.2)$ \\
Died related to ovarian tumor & $1(0.6)$ \\
Died from other causes & $6(3.7)$ \\
5-year progression-free survival (\%) & $93.2(83.7-97.2)$ \\
5-year overall survival (\%) & $97.4(92.1-99.2)$ \\
\hline *7 patients were lost to follow-up &
\end{tabular}

Table 4. Univariable and Multivariable Analyses of 5-Year Progression-Free Survival

\begin{tabular}{|c|c|c|c|c|}
\hline \multirow[t]{3}{*}{ Variables } & \multicolumn{4}{|c|}{ 5-Year Progression-Free Survival } \\
\hline & \multicolumn{2}{|c|}{ Univariable } & \multicolumn{2}{|c|}{ Multivariable } \\
\hline & $\operatorname{HR}(95 \% \mathrm{CI})$ & p-value & aHR $(95 \% \mathrm{CI})$ & p-value \\
\hline Cell type & & 0.48 & & \\
\hline Mucinous & 1 & & & \\
\hline Endometrioid & $5.93(0.54-65.53)$ & & & \\
\hline Serous & $3.09(0.43-21.97)$ & & & \\
\hline Mixed & 0.00 (Not report) & & & \\
\hline Tumor size (cm.) & & 0.03 & & 0.04 \\
\hline$>10$ & 1 & & 1 & \\
\hline$\leq 10$ & $7.67(1.28-46.06)$ & & $9.68(1.17-80.25)$ & \\
\hline Stage & & 0.02 & & 0.01 \\
\hline I & 1 & & 1 & \\
\hline II-III & $8.09(1.35-48.51)$ & & $21.17(2.04-219.75)$ & \\
\hline Primary treatment & & 0.03 & & \\
\hline Definitive surgery & 1 & & & \\
\hline Conservative surgery & $11.08(1.24-99.43)$ & & & \\
\hline Pelvic lymphadenectomy & & 0.38 & & \\
\hline No & 1 & & & \\
\hline Yes & $0.38(0.04-3.37)$ & & & \\
\hline Hysterectomy & & 0.03 & & 0.03 \\
\hline No & 1 & & 1 & \\
\hline Yes & $0.09(0.01-0.77)$ & & $0.07(0.01-0.74)$ & \\
\hline Microinvasion & & 0.95 & & \\
\hline No & 1 & & & \\
\hline Yes & $0.93(0.1-8.3)$ & & & \\
\hline Noninvasive implant & & 0.63 & & \\
\hline No & 1 & & & \\
\hline Yes & $1.71(0.19-15.3)$ & & & \\
\hline Laterality & & 0.07 & & \\
\hline Unilateral & 1 & & & \\
\hline Bilateral & $5.33(0.89-31.9)$ & & & \\
\hline
\end{tabular}


Concerning the oncological outcomes, the 5-year PFS of BOT patients in our study was relatively high at $93.2 \%$ with $1.2 \%$ progression rate to invasive cancer. The previous study reported an approximately 2-3\% progression rate [16]. With the low recurrence rate of BOT in this study $(5.3 \%)$, univariate and multivariate analyses were carried out and showed that advanced stage, tumor size $>10 \mathrm{~cm}$ and not performing hysterectomy were significantly associated with adverse survival outcomes. However, generalizability of these findings may not be applicable. In previous study, the significant prognostic factors were advanced stage, age older than 65 years, and the presence of micro-invasion. In univariate and multivariate analyses, hysterectomy was found to be a significant protective factor for recurrence [14]. This may be due to performing bilateral salpingo-oophorectomy [17]. In a case that does not require fertility, hysterectomy should be considered as a standard of treatment.

Many studies reported an increase of recurrence by conservative surgery, especially ovarian cystectomy $[6,18,19]$. In a meta-analysis, the recurrence rates were frequently noted in patients undergoing ovarian cystectomy, bilateral ovarian cystectomy, unilateral salpingo-oophorectomy (SO), and unilateral SO with contralateral cystectomy accounting for approximately $25.3 \%, 25.6 \%, 12.5 \%$, and $26.1 \%$, respectively. The better outcomes in patients treated with unilateral $\mathrm{SO}$ was observed when compared to those treated with ovarian cystectomy (odds ratio for recurrence reduction $=2.200$, $95 \% \mathrm{CI}=0.793-2.841, \mathrm{p}<0.0001$ ) [20]. However, some studies reported the safety of using conservative surgery $[21,22]$. In our study, conservative surgery was one of the risk factors for recurrence in univariate analysis but was not significant in multivariate analysis. Therefore, conservative surgery could be offered in selected cases in whom the risk should be informed to the patients and long-term follow-up is required to detect tumor recurrence $[23,24]$.

BOT tends to have a relatively large tumor size. The average size of mucinous tumors was approximately 13.0-14.9 $\mathrm{cm}$ larger than that of serous tumors which were 7.2-7.5 $\mathrm{cm}$ [25]. The mean tumor size of BOT in our study was $17.3 \mathrm{~cm}$. Tumor size of larger than $10 \mathrm{~cm}$ was significantly associated with a lower recurrence rate regardless of cell type. Previous study by Chen et al., (2017) reported that a tumor diameter larger than $10 \mathrm{~cm}$ had better PFS (HR 0.26, 95\% CI 0.09-0.70). The strong evidence explaining this correlation remains unknown. Accordingly, patients with larger BOT do not always experience worse oncological outcomes. Therefore, conservative surgery can be offered to younger patients with large tumor size.

The recurrences of BOT in our study were mostly found in patients with advanced stage similar to the previous study [26]. However, the stage of BOT did not significantly affect survival in one study [27]. The existence of non-invasive implantation and micro-invasion did not increase the risk of recurrence $[28,29]$. However, these histologic factors could be controversial for surgeons on oncological outcomes and the selection of extension of surgery, especially micro-invasion. Although, many studies illustrated that micro-invasion increased the risk of recurrence, it did not affect survival outcomes and can be successfully treated by the second operation [30]. Pelvic lymphadenectomy was performed in $37 \%$ of our BOT patients and $3 \%$ had lymph node metastasis. Pelvic lymphadenectomy did not affect the recurrence in univariate analysis. This operation is no longer needed in surgical treatment for BOT [5].

The strength of this study was that all cases were treated in a single institute and the specimens were pathologically reviewed by expert gynecologic pathologists. However, some limitations other than the retrospective nature exist including the short median follow-up time at 25.3 months and a variety of surgical procedures for patients with BOT. Many patients attended later follow-up at other hospitals near home due to public health policy. Therefore, long-term survival outcomes could not be evaluated. The role of tumor marker as preoperative diagnostic methods and adjuvant chemotherapy in BOT remain controversial. These issues were not focused on this research and further study is required to evaluate the precise outcomes.

In conclusion, patients with BOT had an excellent survival outcome. Tumor size larger than $10 \mathrm{~cm}$, early-stage disease, and hysterectomy were significant prognostic factors for better survival. Conservative surgery should be offered to patients who desire to preserve future fertility and long-term follow-up is needed to assure recurrence - free.

\section{Acknowledgments}

The authors wish to thank Faculty of Medicine Research Fund, Chiang Mai University, for the financial support in this study.

\section{Conflict of Interest}

The authors have no conflict of interest to disclose.

\section{Author Contributions}

SP, CT and JS contributed to the literature search, study concepts, study design and data collection. SP, JS, KC and TM contributed to the data analysis and interpretation of data. SP, JS, PS and CT contributed to the drafting of the manuscript. All authors contributed to reviewing and approval of the final version of the manuscript.

\section{References}

1. Hauptmann S, Friedrich K, Redline R, Avril S. Ovarian borderline tumors in the $2014 \mathrm{WHO}$ classification: evolving concepts and diagnostic criteria. Virchows Archiv. 2016 Dec 27;470(2):125-142. https://doi.org/10.1007/s00428016-2040-8

2. Schuurman MS, Timmermans M, van Gorp T, de Vijver KKV, Kruitwagen RFPM, Lemmens VEPP, van der Aa MA. Trends in incidence, treatment and survival of borderline ovarian tumors in the Netherlands: a nationwide analysis. Acta Oncologica. 201905 28;58(7):983-989. https://doi.org/10 $.1080 / 0284186 x .2019 .1619935$ 
3. Skírnisdóttir I, Garmo H, Wilander E, Holmberg L. Borderline ovarian tumors in Sweden 1960-2005: Trends in incidence and age at diagnosis compared to ovarian cancer. International Journal of Cancer. 2008 Oct 15;123(8):18971901. https://doi.org/10.1002/ijc.23724

4. Fischerova D, Zikan M, Dundr P, Cibula D. Diagnosis, Treatment, and Follow-Up of Borderline Ovarian Tumors. The Oncologist. 201209 28;17(12):1515-1533. https://doi. org/10.1634/theoncologist.2012-0139

5. Qian X, Hua X, Wu J, Shen Y, Cheng X, Wan X. Clinical Predictors of Recurrence and Prognostic Value of Lymph Node Involvement in the Serous Borderline Ovarian Tumor. International Journal of Gynecological Cancer. 2018 02;28(2):279-284. https://doi.org/10.1097/ igc.0000000000001154

6. De Iaco P, Ferrero A, Rosati F, Melpignano M, Biglia N, Rolla M, De Aloysio D, Sismondi P. Behaviour of ovarian tumors of low malignant potential treated with conservative surgery. European Journal of Surgical Oncology (EJSO). 2009 06;35(6):643-648. https://doi. org/10.1016/j.ejso.2008.09.011

7. Lee SY, Choi MC, Kwon BR, Jung SG, Park H, Joo WD, Lee C, Lee JH, Lee JM. Oncologic and obstetric outcomes of conservative surgery for borderline ovarian tumors in women of reproductive age. Obstetrics \& Gynecology Science. 2017;60(3):289. https://doi.org/10.5468/ogs.2017.60.3.289

8. Wang Y, Sun H, Yu A, Zhu T, Chen X. Association between chemotherapy and disease-specific survival in women with borderline ovarian tumors: A SEER-based study. European Journal of Obstetrics \& Gynecology and Reproductive Biology. 2019 Nov;242:92-98. https://doi.org/10.1016/j. ejogrb.2019.09.004

9. Ewald-Riegler N, du Bois O, Fisseler-Eckhoff A, Kommoss F, Harter P, Traut A, Hils R, du Bois A. Borderline Tumors of the Ovary: Clinical Course and Prognostic Factors. Onkologie. 2012;35(1-2):28-33. https://doi.org/10.1159/000336140

10. Longacre TA, McKenney JK, Tazelaar HD, Kempson RL, Hendrickson MR. Ovarian Serous Tumors of Low Malignant Potential (Borderline Tumors): outcome-based study of 276 patients with long-term $(>$ or $=5$-year) follow-up. American Journal of Surgical Pathology. 2005 06;29(6):707-723. https://doi.org/10.1097/01.pas.0000164030.82810.db

11. Trimble CL, Kosary C, Trimble EL. Long-Term Survival and Patterns of Care in Women with Ovarian Tumors of Low Malignant Potential. Gynecologic Oncology. 2002 07;86(1):34-37. https://doi.org/10.1006/gyno.2002.6711

12. Jung H, Park J, Kim D, Suh D, Kim J, Kim Y, Kim Y, Nam J. Comparison of Laparoscopic and Open Surgery for Patients With Borderline Ovarian Tumors. International Journal of Gynecologic Cancer. 2018 Nov;28(9):1657-1663. https:// doi.org/10.1097/igc.0000000000001358

13. Khunamornpong S, Settakorn J, Sukpan K, Suprasert P, Siriaunkgul S. Mucinous Tumor of Low Malignant Potential ("Borderline" or "Atypical Proliferative" Tumor) of the Ovary. International Journal of Gynecological Pathology. 2011 05;33(3):218-230. https://doi.org/10.1097/ pgp.0b013e3181fcf01a

14. Song T, Lee Y, Choi CH, Kim T, Lee J, Bae D, Kim B. Risk Factors for Progression to Invasive Carcinoma in Patients With Borderline Ovarian Tumors. International Journal of Gynecologic Cancer. 2014 09;24(7):1206-1214. https://doi. org/10.1097/igc.0000000000000216

15. Song T, Lee Y, Choi CH, Kim T, Lee J, Bae D, Kim B. Histologic distribution of borderline ovarian tumors worldwide: a systematic review. Journal of Gynecologic Oncology. 2013;24(1):44. https://doi.org/10.3802/ jgo.2013.24.1.44

16. Zanetta G, Rota S, Chiari S, Bonazzi C, Bratina G, Mangioni C. Behavior of Borderline Tumors With Particular Interest to Persistence, Recurrence, and Progression to Invasive Carcinoma: A Prospective Study. Journal of Clinical Oncology. 200105 15;19(10):2658-2664. https://doi. org/10.1200/jco.2001.19.10.2658

17. Ouldamer L, Lacoste C, Cormier B, Arbion F, Marret H, Jallais L, Fignon A, Body G. Is there a justification for hysterectomy in patients with borderline ovarian tumors?. Surgical Oncology. 2016 03;25(1):1-5. https://doi. org/10.1016/j.suronc.2015.11.004

18. Helpman L, Yaniv A, Beiner ME, Aviel-Ronen S, Perri T, Ben-Baruch G, Hogen Ben-David L, Jakobson-Setton A, Korach J. Fertility preservation in women with borderline ovarian tumors - how does it impact disease outcome? A cohort study. Acta Obstetricia et Gynecologica Scandinavica. 201709 21;96(11):1300-1306. https://doi.org/10.1111/ aogs. 13203

19. Uzan C, Muller E, Kane A, Rey A, Gouy S, Bendiffallah S, Duvillard P, Fauvet R, Darai E, Morice P. Prognostic factors for recurrence after conservative treatment in a series of 119 patients with stage I serous borderline tumors of the ovary. Annals of Oncology. 2014 01;25(1):166-171. https://doi. org/10.1093/annonc/mdt430

20. Vasconcelos I, de Sousa Mendes M. Conservative surgery in ovarian borderline tumours: A meta-analysis with emphasis on recurrence risk. European Journal of Cancer. 2015 03;51(5):620-631. https://doi.org/10.1016/j. ejca.2015.01.004

21. Chen R, Li J, Zhu T, Yu H, Lu X. Fertility-sparing surgery for young patients with borderline ovarian tumors (BOTs): single institution experience. Journal of Ovarian Research. 201603 18;9(1). https://doi.org/10.1186/s13048-016-0226-y

22. Gungorduk K, Asicioglu O, Braicu E, et al. The Impact of Surgical Staging on the Prognosis of Mucinous Borderline Tumors of the Ovaries: A Multicenter Study. Anticancer Research. 2017 Oct 03;37(10). https://doi.org/10.21873/ anticanres. 11995

23. Tinelli R, Tinelli A, Tinelli FG, Cicinelli E, Malvasi A. Conservative surgery for borderline ovarian tumors: A review. Gynecologic Oncology. 2006 01;100(1):185-191. https://doi.org/10.1016/j.ygyno.2005.09.021

24. Yokoyama Y, Moriya T, Takano T, Shoji T, Takahashi O, Nakahara K, Yamada H, Yaegashi N, Okamura K, Izutsu T, Sugiyama T, Tanaka T, Kurachi H, Sato A, Tase T, Mizunuma H. Clinical outcome and risk factors for recurrence in borderline ovarian tumours. British Journal of Cancer. 200605 09;94(11):1586-1591. https://doi.org/10.1038/ sj.bjc. 6603139

25. Messalli EM, Grauso F, Balbi G, Napolitano A, Seguino E, Torella M. Borderline ovarian tumors: features and controversial aspects. European Journal of Obstetrics \& Gynecology and Reproductive Biology. 2013 03;167(1):8689. https://doi.org/10.1016/j.ejogrb.2012.11.002

26. Chen X, Fang C, Zhu T, Zhang P, Yu A, Wang S. Identification of factors that impact recurrence in patients with borderline ovarian tumors. Journal of Ovarian Research. 201704 04;10(1). https://doi.org/10.1186/s13048-017-0316-5

27. Loizzi V, Selvaggi L, Leone L, Latorre D, Scardigno D, Magazzino F, Cormio G. Borderline epithelial tumors of the ovary: Experience of 55 patients. Oncology Letters. 2014 Dec 02;9(2):912-914. https://doi.org/10.3892/ol.2014.2758

28. Ferrero A, Strada I, Di Marcoberardino B, Maccarini LR, Pozzati F, Rossi M, Biglia N, De Iaco P. Clinical Significance of Microinvasion in Borderline Ovarian Tumors and Its Impact on Surgical Management. International Journal of 
Gynecologic Cancer. 2012 09;22(7):1158-1162. https://doi. org/10.1097/igc.0b013e31825e5254

29. Hogg R, Scurry J, Kim S, Friedlander M, Hacker N. Microinvasion links ovarian serous borderline tumor and grade 1 invasive carcinoma. Gynecologic Oncology. 2007 07;106(1):44-51. https://doi.org/10.1016/j. ygyno.2007.01.054

30. Boyraz G, Salman M, Gultekin M, Ozkan N, Uckan $\mathrm{H}$, Gungorduk K, et al. What is the impact of stromal microinvasion on oncologic outcomes in borderline ovarian tumors? A multicenter case-control study. Arch Gynecol Obstet. 2017;296(5):979-87. https://doi.org/10.1007/ s00404-017-4496-4

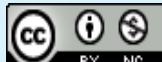

This work is licensed under a Creative Commons AttributionNon Commercial 4.0 International License. 